\title{
LA TEORIA POSTERIOR DE LEIBNIZ SOBRE LA SUSTANCIA MATERIAL
}

Enrique Villanueva

INSTTUUTO DE INVESTIGACIONES FLOSÓficas Universidad Nacional autónoma de Mexico

Por la teoría posterior de Leibniz sobre la sustancia entenderé la teoría que sostiene a partir del año 1695 con el ensayo del Nuevo sistema de la comunicación entre las sustancias, prosigue con los Nouveaux Essais y concluye con los tratados de 1714, principalmente la Monadologia y las Correspondencias con De Volder y con Des Bosses. 1

En otros trabajos me he referido a su teoría de la sustancia de 1686, expuesta principalmente en la Correspondencia con Arnauld. Mi interés principal en este trabajo consiste en explorar su teoría posterior de la sustancia material. Procederé de la siguiente manera: en la primera parte resumiré su teoría de la sustancia como aparece en el Nuevo Sistema y en los Nuevos Ensayos; en la segunda parte expondré su teoría de la sustancia material según aparece en las Correspondencias con De Volder $y$ Des Bosses y señalaré las dificultades que la aquejan; en la tercera parte ofreceré un diagnóstico de la causa de esas dificultades.

Entre la Correspondencia con Arnauld y la Correspondencia con De Volder están dos trabajos importantes de Leibniz, a saber, su tesis del Nuevo sistema de la comunicación entre las sustancias (1695) y los Nouveaux Essais sur l'Entendement Humain (1698-1704). En el primero se enfatiza el concepto de ley como un concepto central para el concepto de sustancia; en el segundo aparecen importantes acotaciones semánticas y epistemológicas que cualifican importantemente el concepto

1 Las citas corresponden a las páginas en la edición de Gerhardt, excepto en los Nouveaux Essais donde los números de las páginas refieren a la paginación de la edición de André Robinet y Heinrich Schepers de 1962 de la Academie Verlag de Berlín. En algunas citas se ofrece con una " $L$ " la paginación de la edición de Loemker de las diversas obras de Leibniz. Finalmente en otros casos no se cita página sino el parágrafo correspondiente. 
de sustancia individual. Me referiré brevemente a esos dos textos importantes que podrán ayudar en lo que viene después.

En el Nuevo sistema (1695) declara que la masa material no es una sustancia $(\S 17)$; en $\S 3$ dice que

es imposible encontrar los principios de la unidad verdadera en la materia sola, o en lo que es meramente pasivo puesto que todo en ella es una colección o acumulación de partes ad infinitum.

Esta concepción de la materia no permite que se la conciba como sustancia. Pero hay otra de acuerdo con la cual la materia tiene leyes del movimiento, esto es, leyes propias, tiene un principio de actividad que es la fuerza (cfr. Explicación del nuevo sistema, § 14). En el $\S 17$ habla de la comunicación entre sustancias corporales.

En la Explicación de las dificultades que encontró Bayle (1696) dice:

Y esta ley del orden, la cual constituye la individualidad de cada sustancia particular, tiene una relación exacta con lo que ocurre en toda otra sustancia...

Es obvio que el cuerpo debe tener una ley de orden pues de otra manera no podría establecerse la correlación legal que exige la armonía. Sin embargo, pronto encontramos a Leibniz diciendo, más adelante en la misma carta, que

lo que es real en la extensión y el movimiento consiste únicamente en el fundamento del orden y la secuencia regulada de los fenómenos y las percepciones.

Y más adelante reafirma que los escépticos encuentran mayores dificultades porque buscan una mayor realidad en las cosas sensibles fuera de nosotros que la realidad de los fenómenos regulados.

En las Observaciones al Articulo 'Rorarious' afirma que:

La materia es un ser incompleto: carece de la fuente original de las acciones.

En los Nuevos Ensayos (1704) vuelve a declarar que los seres por agregación tienen una unidad mental y su ser es mental o fenomenal como el arco iris (146-219). Repite que las cosas sensibles consisten en fenómenos ligados y esta ligazón los distingue de los sueños (374-444). Insiste en que los constituyentes últimos de la realidad son mónadas o unidades perfectas ( $c f r$. p. 440,443,473). La idea extrema es que el 
mundo externo, sensible, en cuanto no es mera ilusión, resulta de las mónadas. Esta superveniencia va a ocasionar los mayores problemas, según veremos posteriormente.

En la introducción a los Nuevos Ensayos afirma, sin embargo, que:

También sostengo que las sustancias, sean materiales o inmateriales, no pueden concebirse en su pura esencia sin ninguna actividad, siendo la actividad la esencia de la sustancia en general.

Más adelante afirma a propósito de la naturaleza corporal:

$Y$ cada vez que encontramos alguna cualidad en un sujeto, debemos pensar que si entendiésemos la naturaleza de este sujeto y de esta cualidad, deberíamos concebir cómo esta cualidad resulta de ese sujeto.

Pero esto sólo lo afirma para concluir:

Así vemos que la materia no tiene naturalmente la atracción mencionada antes, y no va por sí misma en curva, porque no es posible... explicarlo mecánicamente, mientras que lo que es natural debe ser capaz de volverse distintamente concebible si fuésemos admitidos en los secretos de las cosas.

Así pues, aun cuando tampoco aquí se compromete con la existencia de sustancias materiales me parece que su teoría de la sustancia no le impide aceptarlas, pero sus tesis espiritualistas de la inmortalidad, la inmaterialidad del alma, etcétera, lo llevan a ello. Esto es, su rechazo de las sustancias materiales deriva de las necesidades del sistema y de lo que busca garantizar el sistema.

Esta impresión de que su teoría de la sustancia no impide reconocer a los cuerpos materiales como sustancias se ve reforzada por la tesis semántica sobre los nombres de sustancias y clases de sustancias que aparece en los Nuevos Ensayos (III, cap. vi). Leibniz presenta allí una teoría moderada de acuerdo con la cual cuando designamos una sustancia con un nombre de especie generalmente tenemos éxito referencial aun cuando desconocemos en gran medida las propiedades esenciales y accidentales del individuo sustancial o de la clase de sustancia así nombrada. Sabemos que es una unidad, con ley, con entelequia o esencia funcional con ciertas disposiciones pero desconocemos cómo se unifican las propiedades, cómo opera su principio de actividad y cómo modifica las disposiciones de la materia prima, así como cuáles son esas disposiciones. En suma, desconocemos una gran parte de la naturaleza de las 
sustancias pero aun así tenemos la capacidad semántica para hablar inteligiblemente de ellas y finalmente de cómo se relacionan esas disposiciones con la materia segunda. De todas estas cosas fundamentales de la constitución de una sustancia compuesta no tenemos sino la más vaga idea a priori y debemos acumular conocimientos para poder descubrir cómo está constituida.

\section{II}

Llegamos así a la Correspondencia con De Volder que se extiende de 1699 a 1706. Éste es el enunciado más amplio y detallado de su teoría de la materia y los cuerpos materiales. Aun cuando las afirmaciones de Leibniz están formadas en el contexto polémico de eliminar la teoría cartesiana de la materia como extensión, son, sin embargo, lo suficientemente claras para poder comprender su propia teoria filosófica. Especialmente lo es la carta del 20 de junio de 1703 . Leibniz mismo resume su teoría de la siguiente manera:

Distingo por lo tanto: (1) la entelequia o alma primitiva; (2) la materia primera o fuerza pasiva primitiva; (3) la mónada completa formada de las anteriores; (4) la masa o materia segunda o la máquina orgánica en la cual concurren innumerables mónadas subordinadas; y (5) la sustancia animal o corporal que la mónada dominante convierte en una máquina.

Veamos estos cinco constituyentes. De las entelequias dice:

Si hemos de tener seres reales y sustancias, no veo cómo podemos prescindir de unidades verdaderas... no se encontrará unidad verdadera si se elimina la entelequia.

En la carta de marzo 24/abril 3 de 1699 declara que:

no necesitamos buscar otro concepto de poder o fuerza que el del atributo del cual surge el cambio y cuyo sujeto es la sustancia misma.

La entelequia es el principio de actividad de la sustancia toda. Este principio es lo que las teorías constructivistas a la Hume ignoran y por ello no pueden dar cuenta de la unidad real e individualidad e identidad de la sustancia. La entelequia confiere la especifica forma de actividad y desarrollo de la sustancia y es irreductible a cualquier otro elemento. 
Cuando la entelequia se altera o desaparece, la sustancia cambia o se extingue. La cuestión que no aclara Leibniz, sin embargo, concierne a la manera en que esta entelequia lleva a cabo la función unificadora y cómo permite el cambio sin dejar de ser la misma unidad.

De la materia prima dice:

Así, la resistencia de la materia contiene dos factores: impenetrabilidad or Antitipia y resistencia o inercia. Y puesto que esos factores son siempre iguales en un cuerpo o son proporcionales a su extensión, es en ellos que ubico la naturaleza del principio pasivo o de la materia, aun cuando reconozco, en la fuerza activa que se ejerce ella misma en varios modos a través del movimiento, la entelequia primitiva, o en una palabra, algo análogo al alma, cuya naturaleza consiste en cierta ley perpetua de la misma serie de cambios a través de las cuales corre sin impedimento.

La materia prima o fuerza primaria pasiva está constituida por las disposiciones y capacidades constitutivas de la sustancia. Sobre estas capacidades opera la actividad original de la entelequia modificándolas. De la acción de la entelequia y la subsecuente modificación de la materia prima resulta la mónada o individuo sustancial.

Obsérvese que Leibniz dice que la naturaleza de la entelequia es una ley perpetua de una serie de cambios; ¿cómo concebir esta ley como alma? ¿Y cómo interpretarla como una fuerza original?

La idea es que la fuerza o actividad original o entelequia se ejerce, por ejemplo, sobre la antitipia y la inercia y de ello resulta el movimiento (L 517 marzo-abril 1699). Obsérvese que el movimiento es interno a la sustancia pues resulta de la acción de su entelequia sobre su materia prima.

La materia prima siempre está en la mónada (aún en la muerte acompaña al alma, $c f r$. NE, 114) no es divisible ni extensa pero es la fuente de la extensión y el movimiento. Cada sustancia tiene su materia prima que le es peculiar y constituye su individualidad.

De nuevo Leibniz afirma todo esto pero no nos explica cómo acaece la acción de la entelequia sobre la materia prima. Ni cómo surge el ma vimiento y la extensión de la acción de la entelequia sobre la materia prima.

De acuerdo con lo dicho más arriba, de la entelequia y de la fuerza pasiva de la materia resulta la mónada o sustancia corporal. De esta manera quedaría claro que sí hay sustancia material (sólo que ésta no sería la sustancia que concibieron los atomistas o la extensión de Descartes). ¿Qué sucede con la materia segunda? En las líneas que continúan la cita anterior dice Leibniz: 
No podemos dispensarnos con este principio activo o fundamento de la actividad, pues las fuerzas activas accidentales o cambiantes y sus movimientos son ellas mismas ciertas modificaciones de alguna cosa sustancial pero las fuerzas y acciones no pueden ser modificaciones de una cosa meramente pasiva tal como la materia.

Se sigue, entonces, que hay un ser sustancial o primariamente activo que se modifica por una disposición agregada de materia o pasividad. De aquí que las fuerzas secundarias o motoras y el movimiento mismo deba ser atribuido a la materia secundaria o al cuerpo completo que resulta de lo activo y lo pasivo conjuntamente.

Esta última es "la máquina orgánica en la que concurren innumerables mónadas subordinadas". En la carta del 23 de junio de 1699 dice del cuerpo inanimado que:

tiene innumerables entelequias puesto que él consiste a su vez en partes cada una de las cuales está animada o es como si lo estuviera (L 520).

En otra carta, sin fecha pero de 1699, dice:

Puesto que todo cuerpo extenso, como se los encuentra realmente en el mundo, es de hecho como una armada de criaturas, o un rebaño, o una plaza de confluencia, como un queso lleno de gusanos, una conexión entre las partes del cuerpo no es más necesaria que una conexión entre las partes de una armada (L 521).

No hay conexión necesaria entre las partes y unas pueden ser reemplazadas por otras.

La materia segunda es un conjunto organizado de mónadas o individuos sustanciales. Lo que las organiza es una mónada que Leibniz denomina "dominante". Leibniz dice que la mónada dominante convierte en una máquina la masa de mónadas subordinadas que forman la materia segunda.

La materia segunda es el cuerpo orgánico o máquina que cambia continuamente y puede dividirse; como tal, es solamente un agregado sin conexión necesaria entre las partes/mónadas. La materia segunda tiene vis derivada y movimiento.

Formulemos una serie de cuestiones fundamentales que trataremos de responder a partir del texto de la correspondencia.

a) ¿Por qué se introducen entelequias?

¿Cómo opera la entelequia sobre la materia primera? 
b) ¿Por qué no opera la entelequia directamente sobre la materia segunda?

c) ¿Por qué distingue Leibniz entre materia primera y segunda?

d) ¿Cómo opera la materia primera sobre la materia segunda o cómo surge la materia segunda de la materia primera?

e) La nómada (unión de entelequia con materia prima), şe distingue de la materia segunda o máquina orgánica? ¿Cómo se relacionan?

f) ¿La mónada convierte en una máquina (orgánica) a la sustancia animal o corporal (ya constituida previamente como materia segunda) cuando se convierte en mónada dominante? ¿Bajo qué condiciones se convierte la mónada (¿cualquier mónada?) en dominante?

g) ¿La materia segunda es ella misma una sustancia corporal o animal, es decir, una unidad o un agregado solamente? Cuando una mónada se convierte en dominante, ¿quiere decir esto que mediante dicha dominación le confiere auténtica unidad a la sustancia corporal o solamente le confiere un mayor grado de unidad sin llegar realmente a conferirle verdadera unidad?

Para intentar responder a las preguntas anteriores me basaré principalmente en la carta del 20 de junio de 1703.

La respuesta a $(a)$ es como sigue: las entelequias se necesitan porque toda modificación presupone algo permanente, es decir, las fuerzas derivadas que cambian continuamente presuponen algo que permanece. Luego la entelequia es el principio de individuación pues es la ley y el punto de vista (L 530).

Ahora bien, la entelequia la caracteriza como una fuerza o impulso primitivo, una continua fuente de acción que opera contra las potencias de la materia primera, a saber, la impenetrabilidad y la inercia y de la acción del impulso primitivo emergen las fuerzas derivadas del movimiento y la extensión. ${ }^{2}$

La entelequia es una fuerza o impulso permanente que opera en for-

2 Esta manera de exponer la tesis de Leibniz puede llevar a pensar en una lectura reductivista o reduccionista de la sustancia y la identidad. De acuerdo con ella, la sustancialidad de un individuo se reduce a las disposiciones y la entelequia primitiva, cuya acción obedece un orden legal.

Piénsese por otra parte en cierta lectura del principio de la identidad de los indiscernibles que alienta la interpretación reduccionista.

El asunto es complejo y me limitaré a señalar una salida: aun sin echar mano de la simplicidad, creo que la tesis de la entelequia como principio de acción que implica un punto de vista es suficiente para disipar toda sospecha de reduccionismo sin por ello caer en una tesis del sustrato.

Hay otro reduccionismo, a saber, la tesis de reducir toda sustancia a un conglomerado de mónadas; éste solamente está enunciado -quizás forme un proyecto- y no hay indicación de cómo procedería la reducción. (Cfr. p. 50 del presente trabajo). 
ma de ley y en cuanto permanece esta ley puede decirse que permanece la misma sustancia (L 535).

De lo anterior se sigue una respuesta negativa a $(b)$ puesto que la materia segunda es el resultado de la acción de la entelequia sobre la materia primera. Por lo mismo, la materia segunda o masa resultante contiene la entelequia y la materia primera, de otra manera no se entiende cómo puede ser un resultado.

Respecto a (c) Leibniz dice que la materia primera permanece siempre la misma junto con la entelequia, pero que la materia segunda cambia siempre. ¿Cómo entonces puede resultar algo plural, diverso y cambiante de lo que es siempre lo mismo? Leibniz subraya que hay que concebir la materia a la vez como activa y heterogénea si se va a contar con un principio del cambio y con una manera de distinguir los fenómenos (L 529). Parece pensar que hay que introducir la materia segunda o masa concebida como una pluralidad de sustancias. Pero al mismo tiempo desea preservar la unidad y ésta reside en la entelequia. Parece que ambos afanes son incompatibles y por ello la mónada (entelequia + materia primera) debe ser diferente de la materia segunda (masa compuesta de una pluralidad de sustancias que a su vez son mónadas (con entelequia y materia prima) pues de otra manera la masa sería una nada). Pero así yuxtapuestas mónadas y materia segunda no logran ninguna unidad, solamente ejemplifican los dos afanes o propósitos incompatibles:

Dice entonces Leibniz:

Cuando digo que una sustancia aun si es corpórea contiene una infinidad de máquinas, pienso que debe agregarse al mismo tiempo que forma una máquina compuesta de esas máquinas y que además recibe la acción de una entelequia sin lo cual no contaría con un principio de unidad verdadera (L 529).

Parece que este aserto es inofensivo pues lo único que nos dice es que una sustancia corpórea se compone de varias máquinas o unidades o miembros unidos bajo una máquina mayor la cual está regida por una acción regida por una ley o entelequia. La unidad la otorga el encadenamiento conforme a una ley. Pero en el siguiente parágrafo de su carta Leibniz hace un cambio radical:

i) Hablando propia y exactamente quizá no deberíamos decir que la entelequia primitiva impele la masa de su propio cuerpo sino que solamente se combina con una fuerza primitiva pasiva, la cual completa, o con la que forma la mónada. $\mathrm{Ni}$ 
puede [la entelequia] influir las otras entelequias o sustancias que existen en la misma masa.

ii) Pero en los fenómenos o en el agregado que resulta, todo se explica mecánicamente y por ello se entiende que las masas se impelen unas a otras.

iii) En esos fenómenos es necesario considerar solamente fuerzas derivativas, una vez que se ha establecido de dónde surgen esas fuerzas, a saber, los fenómenos o agregados [surgen] de la realidad de las mónadas (L 529).

(i) Leibniz toma en cuenta de su tesis de la no-interacción entre las sustancias y la versión positiva de esa tesis, a saber, la tesis de la expresión. La mónada no influye en las sustancias o mónadas que componen la materia segunda o masa. El lenguaje de la interacción causal no puede usarse aquí. Según (ii) puede hablarse de interacción causal en los fenómenos o entre agregados. Pero luego Leibniz reafirma que (iii) los fenómenos o agregados surgen como productos emergentes de las mónadas y que ellos solamente tienen fuerzas derivativas, no la fuerza original que pertenece solamente a la entelequia. Parece que aún con este cambio sigue existiendo la perplejidad acerca de cómo unifica la mónada a las sustancias subordinadas, o, la que puede ser la misma pregunta, ecómo surgen las fuerzas derivadas de la fuerza original de la entelequia primaria? Es decir, parece que es el resquemor de Leibniz de no permitir la interacción sustancial que lo lleva a concebir la masa como mero fenómeno o agregado y no responde a la pregunta que Arnauld primero, ${ }^{3}$ y De Volder después, le formulan acerca de la unidad sustancial de los cuerpos materiales. Por este camino Leibniz se rehusa a ofrecer una respuesta a la pregunta $(d)$. Luego optará por la tesis de la expresión como respuesta a $(e)$ y finalmente se reducirá a la tesis de los átomos simples espirituales como su concepto de sustancia.

Más adelante, en la misma carta, Leibniz reafirma su antigua intuición:

Considero a la sustancia misma dotada de fuerza primaria activa y pasiva como una mónada indivisible y perfecta - como el ego o algo similar a él- pero no considero de igual manera a las fuerzas derivadas, las cuales se descubre que cambian continuamente (L 530).

El texto parece repetir un requisito que Leibniz impone a las sustancias pero no avanza un ápice en la tarea de explicar cómo esa mónada perfecta dota de unidad a esa variedad cambiante que son los cuerpos

3 Cfr. mi artículo "Sustancia Material en la Correspondencia con Arnaud", en Diänoia, 1983. 
materiales. Así llegamos al parágrafo inmediatamente anterior al que originó toda esta discusión de la correspondencia con De Volder. Allí, precisamente antes de distinguir los cinco componentes de toda sustancia corporal o animal, dice Leibniz:

i) Si piensas en la masa como un agregado que contiene muchas sustancias, todavía puedes concebir una sustancia o entelequia simple pre-eminente en ella.

ii) Además dispongo en la mónada o sustancia simple, completa con una entelequia, solamente una fuerza pasiva primitiva la cual se relaciona con toda la masa del cuerpo orgánico.

iii) Las otras mónadas subordinadas ubicadas en los órganos no forman parte de ella.

iv) Aun cuando ella las requiere inmediatamente.

v) $Y$ ellas se combinan con la mónada primaria para formar la sustancia corporal orgánica, o el animal o la planta (L 530, la separación y numeración es mía).

(i) Reestablece la dificultad de la mónada o entelequia dominante. Una posibilidad consiste en interpretar la dominación como una unidad mayor o más comprehensiva que las unidades particulares. Esto es quizá lo que tiene en mente Leibniz, cuando dice

Para mí nada es permanente en las cosas excepto la ley misma que envuelve una sucesión continua, la cual corresponde, en las cosas individuales, con la ley que determina al mundo todo (L 534).

(ii) Introduce un elemento sorpresivo. Ahora resulta que la materia prima está relacionada con toda la masa del cuerpo orgánico, es decir, que está relacionada con todas las sustancias que componen la masa o materia segunda. Tenemos, entonces, leyes particulares inscritas o incorporadas en una ley más general y una fuerza primitiva positiva que está relacionada con las fuerzas derivadas que resultan de la masa. (Debemos en consecuencia formular la pregunta adicional: ¿Qué relación existe entre fuerza y ley?) Pero, ¿qué tipo de relación guardan entre ambas? Tendremos que esperar a escuchar su polémica con Des Bosses para oír la respuesta de Leibniz, mientras tanto, el espectro de Spinoza se dibuja detrás del cada vez más aparente pluralismo leibniziano.

(iii) Es difícil de interpretar: ¿de qué cosa es de la que no forman parte? ¿De la fuerza pasiva primitiva o de la mónada dominante? En verdad, no pueden formar parte si solamente están coordinadas las sustancias subordinadas con la mónada dominante. Pero parece que Leibniz 
desea una separación radical entre la fuerza pasiva primitiva y las mónadas subordinadas y esto puede deberse a dos razones, a saber, primero, porque, según se apuntó antes, la fuerza pasiva primitiva no cambia, mientras que las fuerzas derivadas que tiene la masa compuesta de mónadas o sustancias sí cambian; y segundo, porque Leibniz no desea la interacción entre las sustancias y las fuerzas derivadas que tienen interacción causal.

Sin embargo, la fuerza pasiva primitiva "requiere inmediatamente" de las sustancias o mónadas subordinadas de acuerdo con (iv). ¿Qué quiere decir esto? ¿Cómo especificar este "requiere inmediatamente"? Puede ser especificada como la coordinación de leyes de que se habló en (i) o como otro caso de la tesis de la expresión, o como se dijo anteriormente se trata de que las fuerzas derivadas resultan de la fuerza pasiva primitiva.

(v) lo que se "combina" son las mónadas subordinadas con la mónada primaria, dominante o pre-eminente para formar la sustancia corporal orgánica. La "combinación" puede interpretarse como la coordinación de (i) que a su vez puede interpretarse como una expresión.

Parece entonces que Leibniz tiene una respuesta a la cuestión de Arnauld y De Volder, a saber, la unidad de la sustancia corporal es la unidad de las leyes de cada sustancia individual incluidas en la ley general que rige el universo. Pero entonces si ésta es la respuesta de Leibniz, uno puede preguntarle por qué si hablamos de una ley del mundo o del universo no podemos en consecuencia hablar de una única y total sustancia (con toda la complejidad interna que deseemos introducirle) y, por lo tanto, ¿por qué cree Leibniz que su teoría de la unidad tiene algo de particular y diferente de la teoría atribuida comúnmente a Spinoza?

Otra cuestión, quizás no diferente de la anterior, consiste en preguntarle cómo va a evitar la interacción entre las sustancias si la naturaleza de éstas consiste en ser órdenes legales de desarrollo y cambio.

Dicho en otras palabras, no parece haber una conexión necesaria entre la tesis de la unidad verdadera y la tesis de los átomos espirituales, por una parte, y la tesis de la no interacción entre las sustancias, por la otra.

Desafortunadamente no puedo perseguir esos problemas aquí. Mi impresión es que Leibniz desea salvarse de caer en una posición como la de Spinoza, apelando a la existencia de múltiples predicados o propiedades (L 532), es decir, de múltiples leyes, pero esto es precisamente lo que está en cuestión.*

* Quizá Leibniz consideró la interacción sustancial como un paso hacia una tesis 
Por otra parte, ya vimos antes cómo pensó en liberarse de la interacción sustancial apelando a la distinción apariencia-realidad (que equipara a la de agregado-unidad). Esta tesis la repite posteriormente diciendo que el cambio puede concebirse como interno a la sustancia (L 531), en cuyo caso no hay interacción; o puede concebirlo como algo externo en los fenómenos, en cuyo caso sí hay interacción.

Mi impresión es que en las cartas posteriores a la del 20 de junio de 1703 hay una lucha continua entre el realismo y el fenomenalismo (L 535 y 537), el monismo y el pluralismo (L 532), la unidad y el agregado (L 534), las fuerzas primitivas y las derivadas ( $L$ 537). La discusión es muy difícil y se vuelve peor debido a que Leibniz pasa de una tesis a otra opuesta con sólo decir "considerando la cuestión cuidadosamente..." (L 537) o bien "propia y exactamente hablando..." (L 539), o "hablando cuidadosamente..." (L 536).

Concluiré mencionando la dificultad que presenta De Volder a Leibniz en una de las últimas cartas, a saber, la del 30 de junio de 1704 . De Volder considera la tesis de que las fuerzas derivadas emergen de las fuerzas primitivas y se queja de que sólo entiende que "emergen". Leibniz replica diciendo que no es así y que De Volder entiende bastante, pues siendo todas las sustancias similares, entendemos en nosotros mismos cómo todas nuestras fuerzas emergen de dos que son primitivas, a saber, la percepción y el apetito y en un cambio súbito afirma:

En verdad, considerando la cuestión cuidadosamente, puede decirse que nada hay en el mundo excepto sustancias simples y en ellas percepción y apetito. La materia y el movimiento, sin embargo, no son tanto sustancias o cosas sino fenómenos de seres percipientes cuya realidad está localizada en la armonía del percipiente consigo mismo (en tiempos diferentes) y con otros seres percipientes (L 537).

No me alarma la tarea de reducir la materia y el movimiento a la percepción y el apetito sino el cambio que hace Leibniz de una cuestión a otra en donde no va a tener menos dificultades.

¿Por qué no dejó Leibniz la cuestión de la "emergencia" como una

spinozista y ello explica su defensa acérrima del atomismo espiritual que le crea todas estas dificultades y otras (cfr. enseguida la tesis del monadum substantiale vinculum). Conviene dejar apuntado desde ahora que el fenomenalismo de Leibniz tiene un origen muy diferente del de Berkeley, a saber, Leibniz declara a la materia un mero fenómeno porque no puede establecer su verdadera unidad (a menos que renuncie a su atomismo espiritual). La cuestión que resta es ésta: ¿por qué no deja Leibniz el monadismo y acepta la forma sustancial como principio de unidad de los individuos? Es decir, ni spinozismo ni fenomenalismo. Véase el temor al spinozismo de Leibniz en la Correspondencia con De Volder. 
cuestión abierta, determinable posteriormente, cuando sepamos más del mundo y sus leyes, en el espíritu de los Nouveaux Essais? ¿Por qué abandona su tesis de la coordinación de leyes? ¿Por qué piensa que el fenomenalismo le ofrece una vía de escape? (Cfr. L 535).

Dicho en otra forma, Leibniz renuncia a ofrecer una respuesta elucidatoria a las preguntas $(f)$ y $(g)$ y prefiere abrazar el monadismo según se verá enseguida.

En la correspondencia con Des Bosses (1709-15), Leibniz vuelve a considerar las cuestiones de la identidad y composición de los cuerpos materiales expresadas en la difícil tesis de la mónada dominante. Las respuestas que ofrece Leibniz sirven también para examinar su doctrina igualmente difícil de la unidad sustancial.

En la carta del 30 de abril de 1709, Leibniz reitera que los cuerpos orgánicos con alma no tienen materia (en el sentido de masa con extensión y resistencia) sino otras entelequias, porque no puede concebirse que en el flujo perpetuo al que se ven sometidos permanezca siempre alguna porción de materia. En cambio

un alma no puede pasar de un cuerpo orgánico a otro sino que permanece siempre en el mismo cuerpo orgánico y ni la muerte puede violar esta ley (L 597).

Más adelante afirma que

no niego alguna unión real metafísica entre el alma y el cuerpo orgánico, de acuerdo con la cual puede decirse que el alma está verdaderamente en el cuerpo... Pero como tal cosa no puede explicarse por los fenómenos y nada cambia en ellos, no puedo explicar más distintamente en qué consiste formalmente esta unión. Basta que esté ligada por la correspondencia (L 598).

La sorpresa inicial se desvanece cuando Leibniz, en la última frase, interpreta la unión como su tesis paralelista de la correspondencia. Leibniz aclara que la unión no se da entre la entelequia y la materia prima -que concedió en la carta a De Volder del 20 de junio de 1703 y de la cual unión resulta la mónada - sino de la unión del alma o mónada que así resulta con la masa o materia segunda que está constituida por otras mónadas.

Para el 5 de febrero de 1712 la posición de Leibniz se torna más clara y aun cuando hay una duda, es claro que, para nuestra sorpresa, piensa introducir una nueva categoría ontológica en su esquema en detrimento de la interpretación de la tesis del espejo o de la expresión. Dice en su carta: 
Si una sustancia corpórea es algo real en adición a las mónadas, como se sabe que una línea es más que dos puntos, tendrá que decirse que la sustancia corpórea consiste en un tipo de unión o más bien en un unificador real [uniente reali] sobreagregado a las mónadas por Dios y que de la unión de la fuerza pasiva de las mónadas surge la materia primaria o la impulsión [exigentia] a la extensión o antitipia o la difusión y la resistencia. De la unión de las ențelequias monádicas, sin embargo, surge una forma sustancial... Tal forma, empero, no será un alma, la cual es una sustancia simple e indivisible (L 600).

La mónada dominante desaparece para dejar su lugar a una forma sustancial que es un unificador real. Pero Leibniz se siente incómodo con esta nueva forma sustancial que no es un alma simple y está en perpetuo flujo y se declara ante un dilema:

o bien los cuerpos son meros fenómenos, en cuyo caso la extensión será también un fenómeno y sólo las mónadas serán reales, pero la unión será suministrada en el fenómeno por la acción del alma percipiente; o si la fe nos constriñe a afirmar sustancias corporales, la sustancia consiste en esa realidad unificadora [realitate unionali] la cual agrega algo absoluto y por lo tanto sustancial, aun cuando fluido, a las cosas que van a unirse (L. ibid.).

Otra manera de poner el dilema consiste en decir que o bien los cuerpos son solamente fenómenos bien fundados, como el arco iris "sueños continuos en perfecto acuerdo unos con otros", o bien se abandona el nivel de la mera congruencia entre mónadas y se afirma una cadena sustancial entre las mónadas, destruyendo con ello su autarquía (el pluralismo atomista) y la tesis complementaria de la expresión. ${ }^{5}$

Éste es el paso fundamental que Leibniz debe dar para reconocerles auténtica realidad a los cuerpos materiales, solamente que parece que este reconocimiento equivale a un cataclísmico reductio ad absurdum de todo el sistema pluralista y espiritualista de Leibniz. La idea misma de la mónada como simple y autárquica debe ser abandonada según lo afirma el propio Leibniz, pues en tal caso ya no serán universos sin contacto; por lo tanto, no habrá expresión y no habrá armonía y no

5 En este dilema Leibniz supone la interpretación fenomenalista del primero de los disyuntos. Pero no tiene que ser así; de acuerdo con la interpretación sugerida a propósito de la correspondencia con De Volder hay que leer fenómeno en el sentido de que es un resultado de la afinidad entre mónadas y no en el sentido de que es algo que aparece sin ser realmente. Parece que Leibniz se confundió en este punto y por ello titubea al comienzo de su correspondencia con Des Bosses. Después reafirmará su posición. 
habrá pura representación, y tampoco habrá simplicidad y, en suma, el paradigma del ego se vendrá abajo.

Desafortunadamente, los textos de Leibniz no son elocuentes en este sentido y no es posible escucharlo sacar una a una las consecuencias de admitir el vínculo sustancial. Por el contrario, los dos tratados publicados al final de su vida, Monadología y principios de la naturaleza y de la gracia reafirman el monadismo.

No obstante el silencio de Leibniz, pueden escucharse cosas interesantes en su diálogo con Des Bosses. Oigamos algunas.

Des Bosses (junio 12, 1712) le propone a Leibniz un modelo que a través de la subordinación de formas sustanciales constituye el individuo caballo con un alma o forma dominante. Leibniz responde (16 junio, 1712) diciendo que:

La unidad de la sustancia corporal en un caballo no surge de ninguna "refracción" de las mónadas sino de una cadena sustancial sobreañadida por medio de la cual nada más cambia en las mónadas mismas.

Leibniz regresa al final de la carta a la tesis de la correspondencia y dice que "no es necesario asumir ninguna cosa fuera de las mónadas o almas". Pero entonces el monadum substantiale vinculum se reduce a la tesis de la expresión de nuevo y Leibniz no abandona el monadismo.

En la carta del 24 de febrero de 1713, Leibniz distingue dos tipos de cadenas sustanciales:

Una cadena sustancial sobreañadida a las mónadas es en mi opinión algo absoluto, tal que aun cuando corresponde exactamente en el curso natural a las afecciones de las mónadas, esto es, a sus percepciones $y$ apetitos, y puede en consecuencia considerarse dentro de la mónada en cuyo cuerpo está su cuerpo; puede, sin embargo, ser independiente de las mónadas en un sentido sobrenatural y puede ser adaptado o removido de otras mónadas aun cuando sus primeras mónadas permanezcan (L 608).

Hasta este punto parece acercarse Leibniz a la tesis realista al hablar de cadenas sustanciales, es decir, siempre y cuando la cadena sustancial no modifique o interactúe con el orden legal interno de la mónada. Un poco más adelante en la misma carta, sentencia:

Pero si se admiten sustancias corporales o cadenas sustanciales, mi opinión deberá ser que ésas están sujetas a la generación y a la corrupción. 
Esto es, no serán auténticos individuos; por lo tanto, hay que rechazar esta teoría.

En la carta del 21 de abril de 1714 Leibniz vuelve a interpretar el monadum substantiale vinculum como su tesis de la expresión y la correspondencia, pues dice que de otra manera las mónadas constitutivas tendrían unidad per accidens únicamente y serían meros fenómenos.

Las cadenas deben sustentarse en seres auténticos y por lo tanto en la armonía entre las mónadas. Luego argumenta:

No creo que admitirás un accidente en dos sujetos al mismo tiempo. Mi juicio acerca de las relaciones es que la paternidad en David es una cosa, el ser hijo en Salomón otra, pero que la relación común a ambas es una mera cosa mental cuya base es la modificación de individuos... (L 609).

Estamos de nueva cuenta en la tesis de que las relaciones son externas: el individuo es ya completo y todo vínculo sustancial debe respetar esto. Leibniz se queda con las mónadas y, de palabra, concede un vínculo entre ellas pero no lo acepta nunca en realidad. Sin embargo, rechaza la posición subjetivista de Berkeley expresamente (marzo 15, 1715) pero este rechazo debe tomarse con cautela pues en la carta citada más arriba equipara la constitución de la sustancia compuesta con la justificación de la realidad de los fenómenos.

Recuérdese sin embargo que según una interpretación de "fenómeno" apuntada a propósito de la Correspondencia con De Volder lo que Leibniz está diciendo no es que los cuerpos materiales sean apariencias sino más bien que metafísicamente hablando, son derivados o secundarios pues la realidad primera es la mónada. Pero si la mónada es la realidad primaria, nunca va a poder lograr la unidad de un cuerpo (es decir, de un conjunto de mónadas). Por lo tanto, la admisión de sustancias corporales con mónada dominante o sin ella implica el rechazo del monadismo.

En la carta del 29 de abril de 1715 Leibniz compara la cadena sustancial a un eco que nada puede cambiar en las leyes de las mónadas pues cualesquier modificacion̂́es que reciba de las mónadas las recibirá, contingentemente, como un eco. En la carta del 19 de agosto del mismo año, Leibniz defiende la legalidad que rige las mónadas y rechaza que se trate de un mecanismo oscuro:

las mónadas extraen cada cosa de sus propios recursos... mediante su mecanismo eminente, para decirlo así, el cual es el fundamento y concentración del mecanismo corporal; de manera que la forma en que el uno se sigue del otro puede explicarse (L 613). 
Y puesto que esto es así, el eco no es superfluo:

un cuerpo que retorna un eco es un principio de acción. Esta cadena será el principio de acción de la sustancia compuesta...

Empero, en vista de lo que ha dicho antes debemos concluir que esto es solamente una manera de hablar pues ese eco no opera por si mismo sino como el reflejo que es de las mónadas que lo componen; si actúa es porque las mónadas tienen el principio de acción; él es solamente un principio derivado, por así decirlo, de acción. ${ }^{6}$

El resumen de todas las tesis de Leibniz aparece en el cuadro sinóptico, suplemento de la carta del 19 de agosto de 1615 a Des Bosses. El cuadro hace ver con claridad que Leibniz nunca se adhiere a la idea del vínculo sustancial; muchas veces parece dudar pero hasta el final mantiene el monadismo, es decir, la pluralidad de átomos espirituales y las tesis concomitantes. En todo caso, Leibniz piensa que el monadismo junto con las tesis de la armonía, la expresión, etcétera, constituye una reconstrucción racional de las intuiciones que sostiene una teoría realista de la sustancia con la idea del vínculo sustancial. Obsérvese que en el cuadro reaparece la tesis de la mónada dominante como principio unificador y con ello vuelven a surgir todas las perplejidades respecto de la tesis reductivista de que un cuerpo se reduce a un conjunto de mónadas. La tesis de la mónada dominante va en el sentido de explicar la unidad de la diversidad de un cuerpo, el monadismo va en el sentido de reducir o diluir el cuerpo en un conjunto de mónadas. Leibniz debe decidir entre una $u$ otra pero parece que desea acomodarse con las dos aun cuando sólo sea verbalmente. (Cfr. Apéndice donde aparece reproducido el cuadro sinóptico de Leibniz.)

\section{III}

El único argumento que Leibniz presenta para rechazar la sustancialidad de los cuerpos materiales es el de la duda acerca de la unidad de esos cuerpos. Pero Leibniz tiene dos tesis de la unidad, una en términos de simplicidad y otra en términos de orden legal. La primera es sumamente oscura y descansa en la doctrina cartesiana del ego. La segunda es compleja pero no hay nada en ella que impida concederle a los cuerpos materiales el status de sustancias (y aun ciertos agregados o compuestos podrian calificar como sustancias).

- Es decir, Leibniz hace una concesión verbal al hablar de monadum substantiale vinculum pero luego procede a darle contenido a esas palabras interpretándolas con su tesis del espejo o la expresión. 
Lo que le impide a Leibniz que declare a los cuerpos materiales sustancias o individuos per se es su doctrina de que la unidad requiere simplicidad. Leibniz ha postulado los simples y cualquier individuo dotado de complejidad quedará por consiguiente privado de carácter sustancial. Esta poderosa intuición de la simplicidad es la que pesa más a la postre en la teoría de Leibniz. ${ }^{7}$ La otra intuición de los individuos auténticos como entes dotados de un principio de acción o entelequia que tienen complejidad pero que esa complejidad se unifica en el propósito o función que cumplen bajo una ley que rige su orden interno, ley que se condice armónicamente con las demás leyes de los demás individuos, la abandona Leibniz.

La tesis de la simplicidad es una tesis a priori, estipulativa, esencialista; la tesis de la unidad legal es una tesis a posteriori, del descubrimiento, de necesidad condicional.

Por otra parte, aun cuando el problema metaf́sico de la individuación tiene una solución radical solamente para un ser infinito que puede aprehender la noción completa de un individuo o su orden legal completo, los seres humanos finitos pueden acceder a los individuos gradualmente mediante sus clasificaciones de especies, las cuales son correctas en general y las vamos conociendo mejor conforme acumulamos información. Pero entonces, dada esta limitación epistemológica, tampoco desde esta perspectiva finita y provisional podemos negar el carácter de sustancias a los cuerpos materiales y a la materia en su conjunto. Ĺsta es la tesis de la sustancia de los Nouveaux Essais, que he resumido críticamente en otro lugar. ${ }^{8}$

Es decir, en el espíritu de los Nouvieaux Essais, al que nos referimos anteriormente, Leibniz debe dejar abierta la cuestión de la unidad sustancial como algo para ser descubierto en el futuro. En lugar de ello estipula que la unidad sustancial tiene que consistir en la simplicidad y concluye, alineándose con los restriccionistas de la identidad Butler y Reid, declarando que solamente el Ego es una sustancia y la conocemos ya. Lo que Leibniz puede decir es que un individuo sustancial debe tener unidad legal pero que la forma que asume esa unidad legal o principio de la acción es algo que debe ser descubierto paulatinamente. Asimismo queda por averiguarse la forma en que la legalidad de cada

7 Según esta interpretación, Leibniz nunca sucumbirá a una posición como la de Spinoza. Para Leibniz tanto el individuo complejo como el cosmos resultan de la legalidad individual de cada mónada y pueden como tales parecer o cambiar sin que por ello cambien o perezcan esas mónadas.

También hay que anotar que Leibniz parece pensar que puede haber dos entidades materiales diferentes y aun opuestas que se condicen por igual con las leyes de las mismas mónadas constituyentes. (Cfr. p. 43, la cita al final de la página.)

8 Cfr. "Sustancias y Especies en los Nouveaux Essais" en Didnoia, 1982. 
mónada contribuye a la legalidad del conjunto, esto es, cómo se relaciona la materia prima con la materia segunda, etcétera. ${ }^{9}$ En suma, Leibniz no debió ceder al apriorismo y, tal como aparece en los Nouveaux Essais, debe decir unas pocas cosas a priori y dejar un conjunto de otras muchas para que se le dé contenido con la información gradual que vamos adquiriendo. ${ }^{10}$

Finalmente, quiero referirme a otro punto en la teớí dé Leibniz que concierne a su posición metafísica en el problema de la reductibilidad o irreductibilidad de la identidad. Leibniz sostiene que un cuerpo material resulta de la afinidad que guardan entre sí otros individuos más básicos ontológicamente hablando. En este sentido. el cuerpo material se reduce —en un sentido leve- a esos individuos más básicos, es decir, el cuerpo material puede dejar de existir pero las mónadas seguirán existiendo. La identidad de un cuerpo ordinario se reduce, así, a la identidad de las mónadas que le permiten existir. Pero hay otras identidades que son ellas mismas irreductibles, a saber, las mónadas (aquí está operando de nuevo la idea de simplicidad como la idea básica). En la reconstrucción racional del cosmos, Leibniz resulta ser un reduccionista de los cuerpos materiales ordinarios y un no-reduccionista de las mónadas o individuos simples. En esta interpretación hay que olvidar lo de la mónada dominante.

En conclusión sugiero que Leibniz tiene una doble teoría de la sustancia. Según la primera teoría, que podemos calificar de platónica y cartesiana, una sustancia es un ente simple, inmutable, imperecedero, irreductible, autónomo, siempre idéntico cuyo únicos ejemplos son los

9 Para un examen somero de algunas dificultades que tiene Leibniz con estas nociones, véase "The Correspondence Between Leibniz and De Volder" de J. J. Russell, reimpreso en Leibniz: Metaphysics and Philosophy of Science. Ed. por R. S. Woolhouse, Oxford, 1981 .

10 Imaginemos esquemáticamente el procedimiento de Leibniz: a partir de ciertas necesidades conceptuales de lo que es un individuo determinamos un conjunto de principios acerca de la naturaleza de ese individuo, como son que está sujeto a la identidad de los indiscernibles, que se rige por una ley, que es un todo en partes, que cae bajo un concepto de especie y ese concepto recoge parte de su naturaleza, que dicha naturaleza es determinable, etcétera. Todo esto constituye, quizás, un argumento trascendental. Lo demás, es decir, la determinación de las sustancias, su natu. raleza, composición, relaciones, etcétera, queda para ser determinada a posteriori.

El procedimiento anterior observa el principio de que la metafísica no puede legislar sobre cuestiones empíricas sino, por el contrario, esperar a que se descubran y acomodarse a esos descubrimientos. En varios lugares Leibniz parece ajustarse al principio anterior. Sin embargo, en otras ocasiones y sobre todo al final de su vida se apartá de él y legisla con el monadismo.

Puede especularse sobre la razón de dicho apartamiento. Quizá se debió a la necesidad de promulgar su propia versión del espiritualismo metaf́sico a la vez en contra de la teoría de Spinoza y de la presión que venía del realismo metafísico abrazado por el Concilio de Trento. 
egos. Todo lo demás son agregados de esos entes simples, que no pueden interactuar entre sí y solamente se expresan unos con otros.

Según la segunda concepción, una sustancia es una unidad determinable, cuya naturaleza vamos descubriendo paulatinamente ${ }^{11}$ a partir del éxito referencial inicial con conceptos de especie o sortals con los que la identificamos; la sustancia es simple o compleja y esto solamente podemos determinarlo con el conocimiento empírico que vamos adquiriendo.

La sustancia es irreductible, es algo sobre y por encima, una estructura perdurable que tiene componentes; el carácter y la relación de esos componentes es algo que también debe determinarse, como, por ejemplo, la relación entre materia prima y segunda o entre la entelequia dominante y las mónadas constitutivas. Pero en las sustancias complejas el todo sustancial no debe pensarse como otra entidad aparte de las entidades constituyentes, sino como enlace de legalidades. Lo que sabemos a priori de la sustancia es que es una unidad legal, que se aviene a conceptos de especie y que su naturaleza y constitución interna es descubrible.

Esta segunda teoría es de inspiración aristotélica; Leibniz se apoya en la primera teoría para rechazar que los cuerpos sean sustancias, pero no hay nada en su segunda teoría que impida conceder sustancialidad a los cuerpos materiales.

11 El criterio del conocimiento gradual podría aplicarse igualmente a la concepción de la sustancia como simple. Podria, pero entonces tenemos que determinar el tipo de simplicidad que está en cuestión. En otro artículo "Las tesis de la unidad sustancial de Leibniz circa 1686" en II Simposio Internacional de Filosofia, México, UNAM, 1985, he sostenido que si la simplicidad se interpreta como legalidad no habrá dificultad de principio; la dificultad surge cuando se dice que la sustancia es simple y se deja indeterminada esta simplicidad, evitando con ello una interpretación realista fuerte o trascendente. En este último caso no se comprende lo que sería descubrir la sustancia y tampoco lo que constituiría un conocimiento gradual de la misma. 


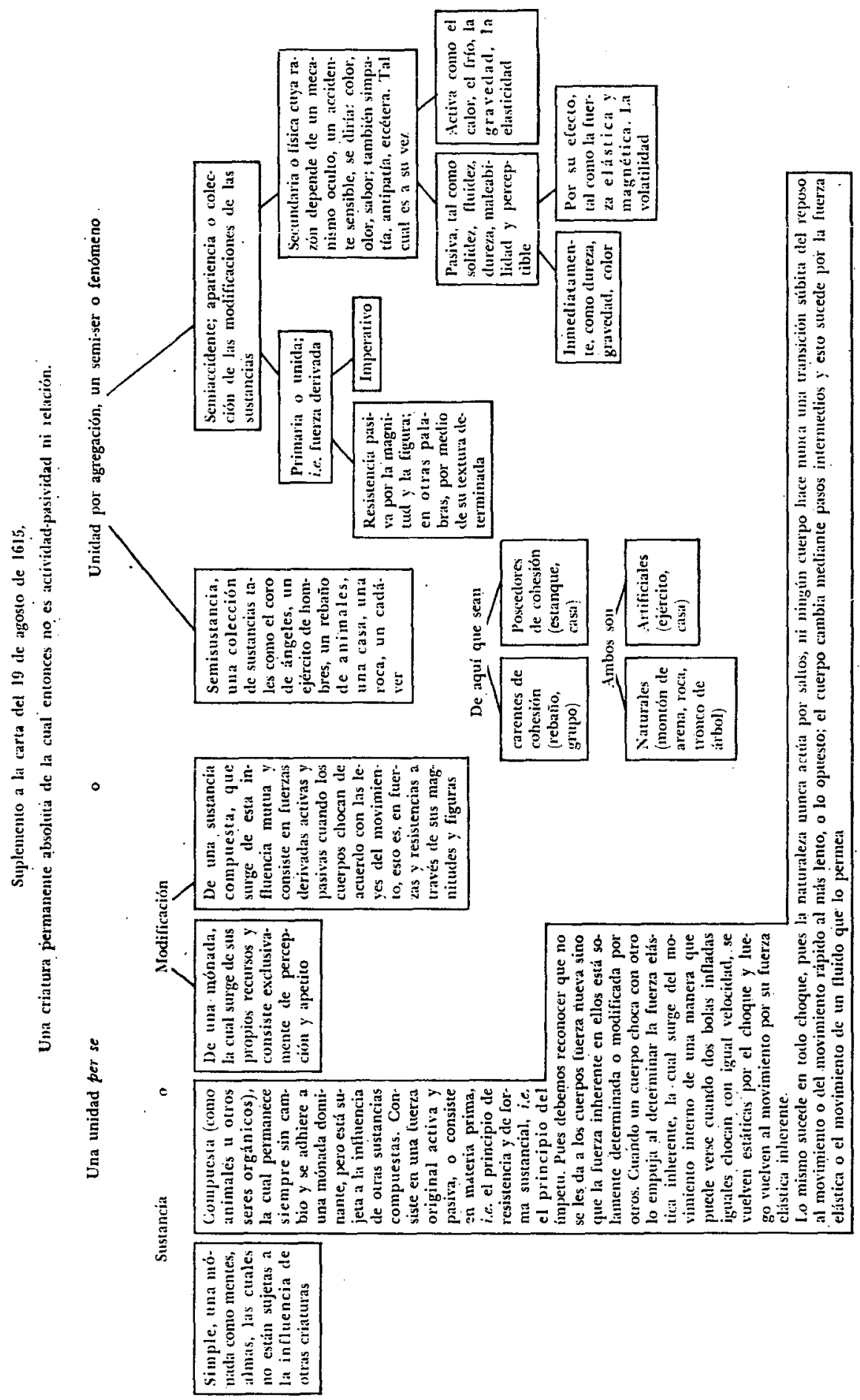

